ARTICLE

\title{
Première mention de Nomada barcelonensis COCKERELL, 1917 pour la France (Hymenoptera : Apidae : Nomadinae)
}

\author{
Éric GABIOT ${ }^{1}$ (D) . Éric DUFRÊNE ${ }^{2}$ (1)
}

GABIOT, É. \&É. Dufrêne (2021). Première mention de Nomada barcelonensis COCKERELL, 1917 pour la France (Hymenoptera: Apidae : Nomadinae: Nomada). Osmia, 9: 83-88. https://doi.org/10.47446/OSMIA9.11

\begin{abstract}
Résumé
Les auteurs relatent ici la découverte au sein de la frange méditerranéenne de la France métropolitaine, dans le département du Var, d'une espèce de Nomada jusqu'alors connue uniquement d'Espagne : Nomada barcelonensis CoCKERELL, 1917. Le statut systématique, la description morphologique et la biologie permettant sa détermination sont présentées ainsi que les conditions de capture du spécimen.
\end{abstract}

Mots-clefs $\mid$ abeille $\cdot \operatorname{Var} \cdot$ nouvelle espèce

First mention of Nomada barcelonensis COCKERELL, 1917 for France (Hymenoptera: Apidae: Nomadinae)

\section{Abstract}

The authors relate here the discovery within Mediterranean fringe of metropolitan France, in the Var department, of a species of Nomada so far only known from Spain: Nomada barcelonensis COCKERELL,1917. The ecology, biology and morphological description allowing its determination are presented as well as the conditions of capture of the specimen.

Keywords | bee $\cdot \operatorname{Var} \cdot$ new species

Reçu•Received | 28 June 2021 || Accepté · Accepted | 14 December 2021 || Publié (en ligne) • Published (online) | 30 December 2021 Reviewers | K. LOUADI • V. LECLERCQ || http://zoobank.org/CA623771-9E18-4486-900B-79C55A1188B2

\section{INTRODUCTION}

Les nomades (Hymenoptera : Apoidea : Nomadinae : Nomadini : genre Nomada SCOPOLI, 1770), abeilles sauvages clepto-parasites, pondent leurs œufs dans le nid d'autres abeilles. Elles parasitent uniquement les abeilles terricoles et principalement les andrènes mais aussi les panurges, halictes, eucères voire les mélittes. Généralement actives de mars à septembre, la faune printanière est, de loin, la plus diversifiée et la plus abondante. La plupart des espèces sont univoltines, i.e. avec une génération par an. Certaines espèces, minoritaires, sont bivoltines voire trivoltines ou quadrivoltines (Nomada).

De nombreux travaux dont ceux de NOBILE (1990), SCHEUCHL (2000) et SMIT (2014) ont éclaircis la connaissance et la compréhension de l'écologie des nomades. Les Nomada recherchent les endroits ensoleillés et riches en fleurs dont elles prélèvent le nectar, elles sont dites polylectiques car butinant sur toutes les sortes de fleurs. On les trouve dans une grande variété d'habitats : chemins, prairies, landes, dunes, friches, carrières, bocage, jardins... à l'exception des milieux trop humides, trop fermés ou trop ventés.

On recense 105 espèces de nomades en France (SMIT, 2018; SCHWARZ \& SMIT, 2020). Une nouvelle sous-espèce, Nomada numida ssp. numida LEPELETIER, 1841 a été découverte depuis (GABIOT \& DUFRÊNE 2018). La faune française compte désormais 106 espèces avec cette découverte.

\section{OBSERVATIONS SUR LE TERRAIN}

Située sur les hauteurs de la commune du Beausset (figure 1) dans le département du Var, la Maison départementale de la nature dite des « Quatre Frères " (https://www.facebook.com/MDN4freres/) (43,225315N,

1 [EG] Société des Sciences Naturelles et d'Archéologie de Toulon et du Var, 2 allée Amiral Courbet, F - 83000 Toulon, France•gabiot.eric@gmail.com - https://www.ssnatv.fr

(i) https://orcid.org/0000-0002-8429-9104 • zoo:ank http://zoobank.org/0D555FDA-69B6-44B0-94B2-A799D8BE2AF0

2 [ED] Observatoire des Abeilles, 9 allée des Pins, Malassis, F - 91470 Forges-les-Bains, France • eric.dfn@wanadoo.fr • https://oabeilles.net/ (iD) https://orcid.org/0000-0001-8897-5512 • zoosank http://zoobank.org/15C8BE99-4FE9-4CF8-ADDE-0716C23F7417 


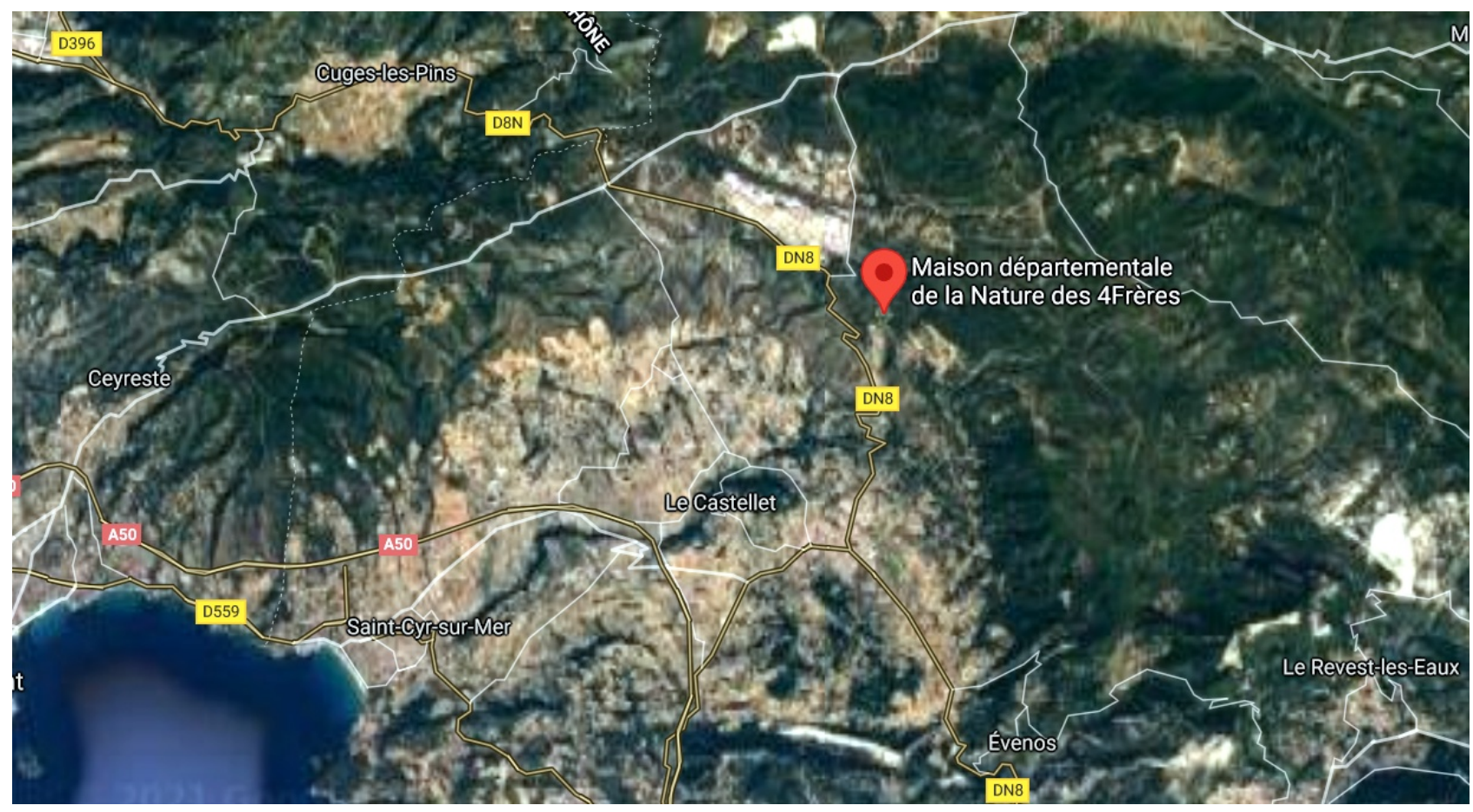

Figure 1. Localisation de la Maison de la Nature des Quatre Frères. Image Google Maps.

$5,813498 \mathrm{E}$ ) (figure 2) reçoit régulièrement le groupe entomologie de la Société des Sciences Naturelles et d'Archéologie de Toulon et du Var (SSNATV). Ce groupe a notamment pour objectif de dresser un inventaire entomologique du site le plus exhaustif possible et prospecte à cette fin depuis 2016 l'ensemble de ces milieux, en s'attachant à multiplier les sorties sur l'ensemble des saisons.

On peut dresser le bilan suivant :

- Deux visites ont été faites en 2016 (avril et septembre).
- Trois en 2017 (avril, juin et septembre).

- Deux en 2018 (juin et octobre).

- Trois en 2019 (mai, juillet, octobre).

La richesse entomologique du lieu des Quatre Frères tient à la diversité de ses biotopes (garrigues, pelouses sèches, prairies fleuries...) et à leur gestion (absence de pesticide, fauchage raisonné...). L'entrée du site est composée de plusieurs restanques en zone sèche, non cultivées et dont la forte proportion de zones de sols nus est propice à la

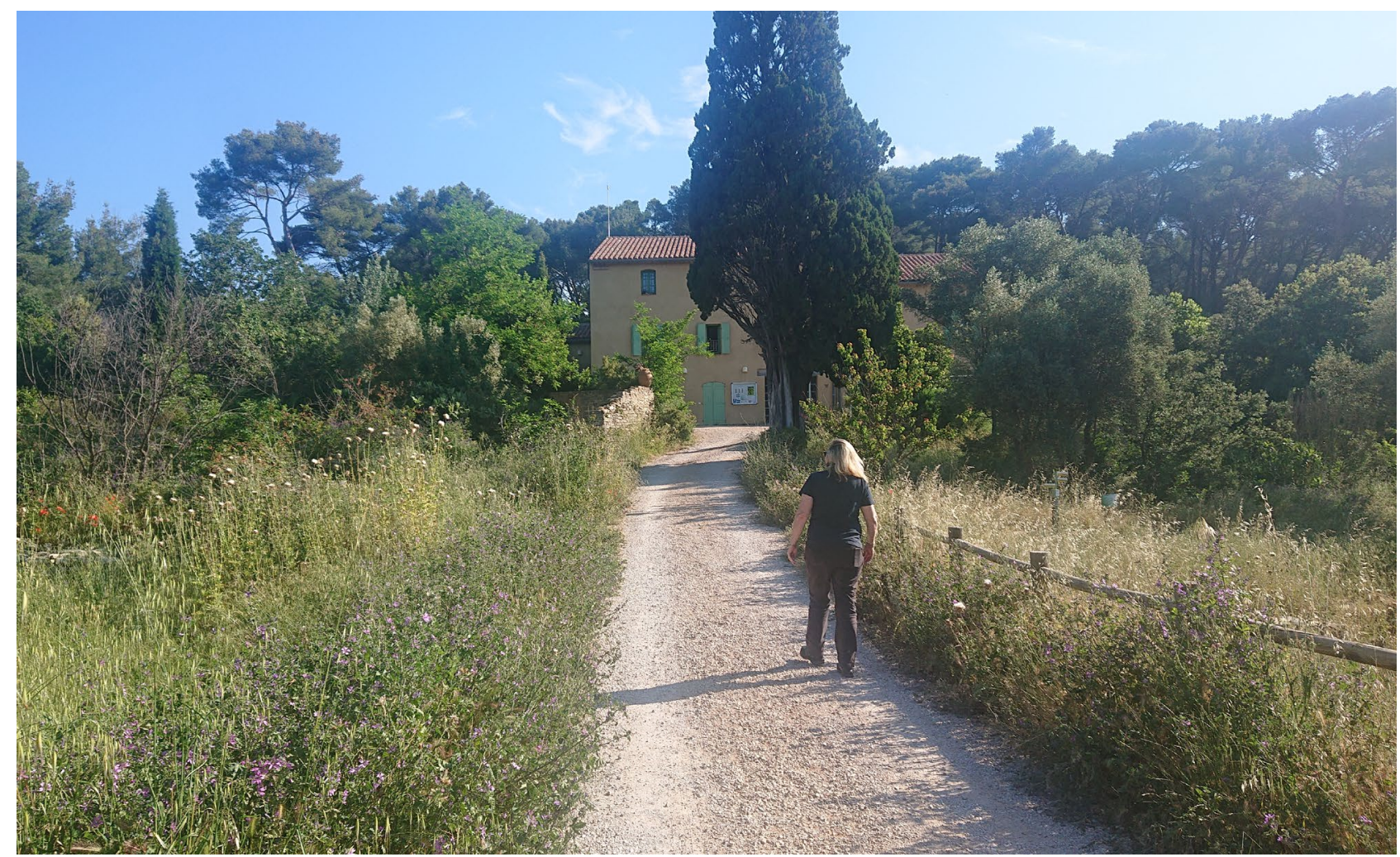

Figure 2. Maison de la nature des Quatre Frères (Le Beausset, France). Cliché É. GABIOT. 


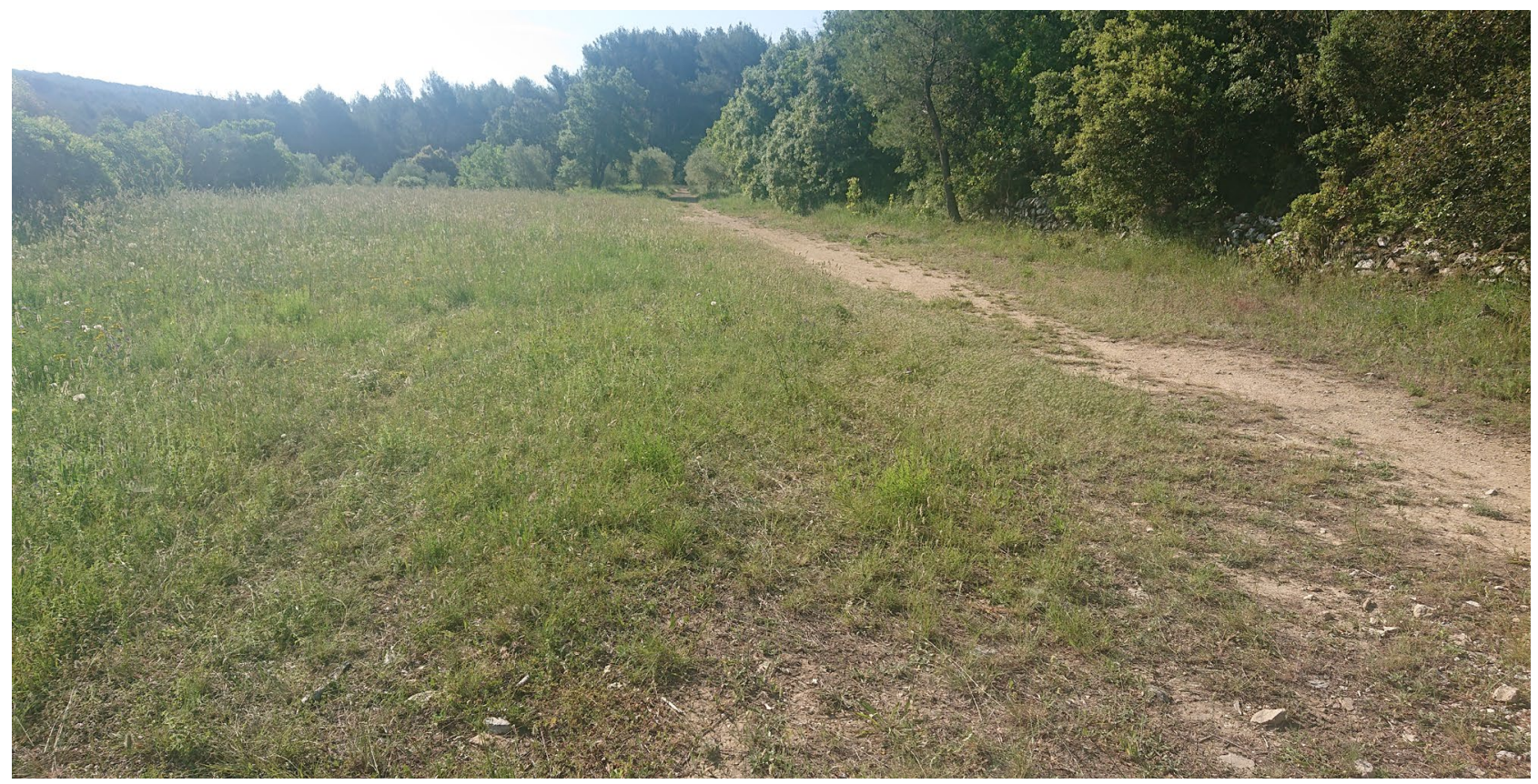

Figure 3. Lieu de capture de Nomada barcelonensis COCKERELL, 1917. Cliché É. GABIOT.

nidification de nombreux individus. Ainsi, une diversité importante d'abeilles sauvages a été collectée en abondance sur ces milieux. Jusqu'à ce jour, 82 espèces d'abeilles ont pu être identifiées sur ce site.

La femelle de Nomada barcelonensis COCKERELL 1917 a été capturée le 30 mai 2019 au filet, sur une des restanques dont le cortège floristique était abondant et typique de la basse Provence calcaire (cardère sauvage, mélilot blanc, millepertuis calycinal, verveine officinale, romarin, lavande...) (figure 3). Plusieurs mâles et femelles d'eucères ont pu être observés sur les mêmes fleurs. C'est à cette occasion et faisant suite à la capture d'une femelle d'Eucera nigrescens PÉREZ, 1879 et une autre d'Eucera longicornis (LINNAEUS, 1758) qu'une nomade a été capturée. Après examen à la binoculaire, celle-ci s'est avérée être Nomada barcelonensis COCKERELL 1917 (É. DUfRÊNE dét.).

\section{STATUT SYSTÉMATIQUE}

\section{Nomada barcelonensis COCKERELL, 1917}

Nomada excellens Pérez, 1913 (nec COCKERELL, 1903) : 323-325 (description Oे, O). « Barcelone », (MNHN). DUSMET, 1913: 254-256

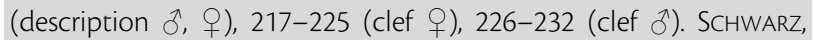
1975 : 118 (désignation des types, lectotype + , allolectotype ô). $^{\text {) }}$.

Nomada barcelonensis COCKERELL, 1917: 237 (nouvelle combinaison).

Nomada barcelonensis montarcoi (DUSMET, 1935) : 49 (sexe non précisé), nomen nudum. «Montarco y Vaciamadrid». CSIC. ALEXANDER \& SCHWARZ, 1994 : 245 sic montarcosi (catalogue). AlbalaDejO \& MOYA, 2006 : 125, sic montarcosi (deux syntypes, sexe non précisé).

L'espèce a d'abord été décrite par PÉREZ en 1913 sous le nom de Nomada excellens, sur la base de spécimens collectés à Barcelone en mai et juin. PÉREZ ne donne pas la localisation précise des spécimens ayant servi à la description, ni le nom de celui qui les a récoltés, ni où ils furent mis en collection. DUSMET redonne une description du mâle et de la femelle de l'espèce en 1913 dans sa révision de la faune espagnole des nomades, sur la base de cinq femelles et quatre mâles issus de la collection de Josep Maria BOFILL I PICHOT. Ces spécimens sont probablement conservés au «Museu de Ciències Naturals de Barcelona ». Il s'agirait, selon DUSMET, du même lot de spécimens que ceux ayant servi à $M$. PÉREZ à décrire l'espèce, d'autant plus que PÉREZ et BOFILL auraient collaboré à ce sujet (DUSMET, 1913, 1920). SCHWARZ désigne ensuite en
1975, dans le cadre de ses travaux de révision des types des espèces de nomades décrites par PÉREZ, un lectotype femelle, un paralectotype femelle et un allolectotype mâle, au sein de la collection de l'auteur au MNHN de Paris.

COCKERELL avait cependant décrit en 1903 une espèce de nomade sous le nom de Nomada excellens et a donc renommée en 1917 l'espèce décrite par PÉREZ sous le nom de Nomada barcelonensis.

DUSMET a par ailleurs cité dans un compte-rendu de ses expéditions sur la péninsule ibérique paru en 1935, la sousespèce Nomada excellens montarcoi, sur la base de spécimens récoltés à Montarco, mais sans localité précise, sans description ni précision des sexes. Ce taxon est listé dans le catalogue d'ALEXANDER \& SCHWARZ publié en 1994, mais demeure absente de la liste des abeilles d'Espagne établis par ORTIZ-SÁNCHEZ (ORTIZ-SÁNCHEZ, 2011, 2020).

Il pourrait être intéressant de préciser le statut taxonomique de cette sous-espèce grâce à l'examen des types situés au muséum des sciences naturelles de Madrid (MARTín ALBALADEJO \& IZQUIERDO MOYA, 2006).

Jusqu'à ce jour, Nomada barcelonensis n'était connue que d'Espagne et donc considéré comme une espèce endémique. 


\section{MORPHOLOGIE}

L'espèce est morphologiquement très proche de Nomada gredosiana SCHWARZ \& GUSENLEITNER, 2013. Des critères morphologiques et des illustrations pour séparer les mâles et les femelles de $N$. barcelonensis et de $N$. gredosiana sont proposés par SCHWARZ \& GUSENLEITNER (2013), constituant d'utiles compléments à la monographie de SMIT (2018).

\section{Le spécimen capturé dans le Var}

Le spécimen collecté est une femelle. La tête est majoritairement noire. Les mandibules sauf l'apex, le labre, les deux tiers apicaux du clypeus et une ligne bordant l'œil composé de chaque côté sont rouges. Les antennes sont rouges, sauf l'arrière des trois premiers articles qui est taché de noir.

Le thorax est majoritairement noir. Le pronotum et les calli sont jaunes. Les tegulae, le scutellum, le postscutellum et deux grandes taches sur les mésopleures sont rouges. Les pattes sont rouges, sauf les coxaes postérieures qui sont partiellement noires. L'abdomen est rouge avec trois taches noires sur le tergite 1, une tache noire à la base du 4 et une bande noire à la base du 5 .

Le labre possède une carène subapicale horizontale avec une dent centrale prolongée verticalement vers le centre du labre par une petite carène longitudinale se terminant par une dent.

L'abdomen est rouge. Le segment antennaire 3 est aussi long que le 4. Le tibia postérieur présente une faible invagination à l'apex portant un petit poil en son centre, ce critère étant typique de l'espèce (SMIT, 2018).

\section{BIOLOGIE ET RELATION HÔTE-PARASITE}

Les espèces parasitées par Nomada barcelonensis COCKERELL 1917 ne sont pas connues. Cependant, plusieurs Eucera longicornis (LINNAEUS, 1758) et Eucera nigrescens PÉREZ, 1879 ont été relevées au même endroit que le prélèvement de la Nomada (à défaut d'autres espèces potentiellement parasitées comme les Andrena). II se peut donc que les Eucera fassent partie des espèces parasitées par Nomada barcelonensis. D'autres relevés sur le terrain devront être réalisés pour identifier la ou les espèces hôtes.

\section{DISCUSSION ET CONCLUSION}

En raison de sa grande taille ( 9 à $13 \mathrm{~mm}$ ) et de l'éloignement relatif des stations espagnoles, la découverte de cette espèce dans le Var peut paraître surprenante. Quoi qu'il en soit, cette découverte confirme la nécessité de poursuivre les inventaires en cours dans le Var et plus largement sur l'ensemble de la façade méditerranéenne afin de contribuer à l'amélioration de notre connaissance régionale de notre apidofaune.
Des prospections et observations complémentaires futures sur ce même site pourraient permettre d'approfondir nos connaissances sur la biologie de Nomada barcelonensis et de découvrir son ou ses espèces hôtes. L'ensemble de la façade méditerranéenne mériterait d'être prospectée pour préciser la répartition géographique de l'espèce. 


\section{RÉFÉRENCES}

AleXander, B. A. \& M. Schwarz (1994). A catalog of the species of Nomada (Hymenoptera Apoidea) of the world. The University of Kansas Science Bulletin, 55(7): 239-270.

Amiet, F., M. Hermann, A. Müller \& R. Neumeyer (2007). Fauna Helvetica 20. Apidae 5: Ammobates, Ammobatoides, Anthophora, Biastes, Ceratina, Dasypoda, Epeoloides, Epeolus, Eucera, Macropis, Melitta, Nomada, Pasties, Tetralonia, Thyreus, Xylocopa. Centre Suisse de Cartographie de la Faune (CSCF) Neuchâtel (Switzerland), 356 pp

BOFILL Y PICHOT, J. M. A. (1904-1905). Catàlech de Insectes de Catalunya. Hymenôpters. XIX Familia. Apidae. Institució Catalana d'Història Natural, Barcelona (Spain), 75 pp. https://doi.org/10.5962/bhl.title.11536

Ceballos, G. (1956). Catálogo de los Himenópteros de España. Consejo Superior de Investigaciones Científicas, Instituto Español de Entomología, Madrid, 554 pp.

CoCKereLL, T. D. A. (1903). Bees of the Genus Nomada from California. Proceedings of the Academy of Natural Sciences of Philadelphia, $\mathbf{5 5}$ 559-579. https://www.jstor.org/stable/4062914

CockerelL, T. D. A. (1917). Descriptions and Records of Bees. The Annals of Magazine of Natural History, 8(20): 235-241. https://doi.org/10.1080/00222931709486998

Dufrêne, É., M. SCHWARZ \& J. SMIT (2014). Le genre Nomada SCOPOLI en France continentale et en Corse : citation de 15 espèces nouvelles pour la faune de France et mise à jour de la liste taxonomique des espèces (Hymenoptera: Apoidea, Anthophila). Linzer biologische Beitrage, 46(2): 1479-1490.

https://www.zobodat.at/pdf/LBB_0046_2_1479-1490.pdf [accessed 15 June 2021]

Dusmet y Alonso, J. M. (1913). Los Ápidos de España. IV - Gen. Nomada FAB. Memorias de la Real Sociedad Española de Historia Natural, 9(2): 203-395

DUSMET Y ALONSO, J. M. (1920). Observaciones sobre la procedencia Espanola de muchos Ápidos descritos por Pérez en "Espèces nouvelles de mellifères de Barbarie". Boletín de la Real Sociedad Española de Historia Natural, 20: 168-169.

DUSMET, J. M. (1935). Cuarenta y cinco años en busca de himenópteros en España. Excursiones, consejos y resultados. Memorias de la Sociedad Entomologica de España, 4: 1-116.

GABIOT, É. \& É. DUfrêne (2018). Première mention de Nomada numida Lepeletier 1841 (Apoidea - Apidae - Nomadini) pour la France continentale. Osmia, 7: 5-9. https://doi.org/10.47446/OSMIA7.1

Martín Albaladejo, C. \& I. IzQuierdo Moya (2006). Tipos de especies de insectos descritas de la comunidad de Madrid, conservados en el Museo Nacional de Ciencias Naturales. Inventario preliminar. Graellsia, 62 (número extraordinario): 109-144. https://graellsia.revistas.csic.es/index.php/graellsia/article/view/11 7/116 [accessed 15 June 2021]

OrTIZ-SÁNCHEZ, F. J. (2011). Lista actualizada de las especies de abejas de España (Hymenoptera: Apoidea: Apiformes). Boletín de la Sociedad Entomológica Aragonesa, 49: 265-281.

http://www.sea-

entomologia.org/Publicaciones/PDF/BOLN_49/265281BSEA49C atalogoAbejas.pdf [accessed 15 June 2021]

Ortiz-SÁnChEZ, F.J. (2020). Checklist de Fauna Ibérica. Serie Anthophila (Insecta: Hymenoptera: Apoidea) en la península ibérica e islas Baleares (edición 2020). In: M. A. RAmOS \& M. SÁNCHEZ Ruiz (ed.) Documentos Fauna Ibérica 14. Museo Nacional de Ciencias Naturales, CSIC, Madrid, ii + 85 pp.

http://www.fauna-iberica.mncn.csic.es/publicaciones/dfi/dfi0014.pdf [accessed 15 June 2021]

PÉreZ, J. (1913). Quelques Nomades d'Espagne nouvelles ou mal connues. Boletín de la Real Sociedad Espanola de Historia Natural, 13: $323-335$

Rasmont, P., D. Genoud, S. Gadoum, M. Aubert, E. Dufrêne, G. Le Goff, G. MAHÉ, D. Michez \& A. PAUlY, (2017). Hymenoptera Apoidea Gallica : Liste des abeilles sauvages de Belgique, France, Luxembourg et Suisse. Atlas Hymenoptera, Université de Mons, Mons, Belgium, 15 pp. http://applications.umons.ac.be/docnum/c7b423fd-d183486c-9cec-966066b9b364/611CA728-5E70-4A5C-8743247E54A8D46F/414_Rasmont_et_al_2017_Hymenoptera_Apoi dea_Gallica_2017_02_16.pdf [accessed 15 June 2021]

SCHWARZ, M. (1975). Ergebnisse der Untersuchungen der von J. Pérez 1913 im Bol. de la Real. Soc. Esp. de Hist. Nat. beschriebenen Nomada-Arten (Hymenoptera, Apoidaea). Nachrichtenblatt der Bayerischen Entomologen, 24: 118-125.

SCHWARZ, M. \& F. GUSEnLeITNER (2013). Zur Kenntnis der NomadaFauna Spaniens mit Klärung der Nomada dolosa MocsáRY (= Nomada centenarii DUSMET) (Hymenoptera: Apidae). Linzer biologische Beiträge, 45(1): 971-993.

https://www.zobodat.at/pdf/LBB_0045_1_0971-0993.pdf

[accessed 15 June 2021]

SChWARZ, M. \& J. SMit (2020). Fünf neue Nomada-Arten aus der WestPaläarktis (Hymenoptera, Apidae). Linzer biologische Beiträge, 52(1): 683-694. https://www.zobodat.at/pdf/LBB_0052_1_0683-0694.pdf [accessed 15 June 2021]

SMIT, J. (2018). Identification key to the European species of the bee genus Nomada SCOPOLI, 1770 (Hymenoptera, Apidae), including 23 new species. Entomofauna, Monographie 3: 1-253. https://www.zobodat.at/pdf/ENT_M3_0001-0253.pdf [accessed 15 June 2021]
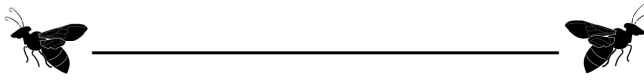
OSMIA est éditée par I'Observatoire des Abeilles (OA), une association loi 1901 d'apidologues (ou mellitologues) d'Europe francophone qui œeuvrent pour la connaissance et la protection des Abeilles sauvages

Les articles sont :

- publiés uniquement en ligne

- disponibles en open access.

- indexés / archivés par Crossref. Zoobank, HAL, Zenodo OpenAIRE, Google Scholar et Web of Science (Clarivate) OpenAIRE, Google
[Zoological Record]

- respectueux des recommandations de la Commission

internationale de Nomenclature zoologique (ICZN),

sous Licence Creative Commons Attribution International CC BY 4.0 qui autorise la reproduction et la diffusion du document, à condition d'en citer explicitement la source

- librement déposables sur des sites internet ou des plateformes d'archivage.

(!) Les documents d'autres sources et non distribuées sou licence libre sont reproduits après autorisation (à demander par les auteurs) et demeurent la propriété des auteurs ou éditeurs originaux.

(!) Le contenu publié est sous l'entière responsabilité des (!) Le conte
auteurs.

OSMIA est conçue pour une impression recto-verso en haute résolution. Les bibliothèques publiques, les laboratoires, les muséums et les associations sont invités à imprimer et conserver une version papier de la revue.
OSMIA is published by the Observatory of Bees (OA), a nonprofit society of apidologists (or mellitologists) from Frenchspeaking Europe who work together for the knowledge and protection of wild bees.

The items are:

- published only online

- available in open access

- indexed / archived by Crossref, Zoobank, HAL, Zenodo, OpenAIRE, Google Scholar and Web of Science (Clarivate) OpenAIRE, Google Sch

- respectful of the recommendations of the International Commission for Zoological Nomenclature (ICZN)

under Creative Commons Attribution Licence International CC BY 4.0 which authorises the reproduction and distribution of the document, provided the source is explicitly cited

- freely depositable on personal or institutional websites and archiving platforms.

(!) Documents from other sources and not distributed under a free license are reproduced after authorisation (to be requested by the authors) and remain the property of the original authors or publishers

(!) The published content is the sole responsibility of the (!) The

OSMIA is designed for high-resolution printing on both sides. Public libraries, laboratories, museums, and societies are invited to print and keep a paper version of the journal.
Directeur de la publication - Editor-in-chief Benoît GESLIN

Comité éditorial • Editorial Board

Matthieu AUBERT • Floriane FLACHER • Mehdi ISSERTES

Tanguy JEAN • Léa LEMAIRE

Mise en page $\bullet$ Layout

Mehdi ISSERTES • Tanguy JEAN • Léa LEMAIRE

Comité de lecture • Scientific committee 2021 https://www.osmia-journal-hymenoptera.com/equipe-team.html

Soumission d'articles • Submission of items osmia.editor@gmail.com

Recommandations aux auteurs Recommendations to authors https://www.osmia-journal-hymenoptera.com/auteurs-authorsinstructions.html

Observatoire des Abeilles

68 rue du Onze Novembre

F - 59148 Flines-lez-Râches (France)

https://oabeilles.net/

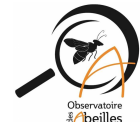

OPEN 